FWS: JHMC

$\mathrm{V}-2$
DEPARTMENT OF COMMERCE

BUREAU OF STANDARDS

WASHINGTON, D. C.

August I, 1932.
Letter

Circular

LC 339

\title{
FLOOR OILS
}

This letter circular has been prepared as a result of the numerous requests received by the Bureau of Standards for general information regarding floor oils.

A mixture of one part of boiled linseed oil (a drying oil) and three parts of turpentine has been used as a floor oil. However, it is our understanding that the floor oil in common use is a well-refined petroleum oil with a flash point not lower than $275^{\circ} \mathrm{F}$, such as paraffin oil. These oils are nondrying oils, are often scented or perfumed and sometimes colored.

After washing a floor it should be allowed to dry before oil is applied. The time of drying will depend upon the kind of wood, the degree of wetness, and the atmospheric conditions. It would seem that a floor might be oiled the same day it is washed, provided it is wiped dry and the weather conditions and ventilation facilities are favorable for rapid evaporation of moisture. However, it would probably be safer to wait until the next day before applying the oil. After the oil is applied it should be rubbed off until only a thin film remains, in order that the floor may not be too slippery. However, some woods may absorbthe oil more readily than others and not require subsequent rubbing.

The oiling of floors is often objectionable in that the oil tends to hold dust and dirt. Floor oil is not in general use in Government Establishments although some branches of the Government have used it, but we are unable to advise as to the extent of such use.

It is believed that waxing is the most satisfactory treatment for wood floors. The wax may be applied to the clean floor that has been previously treated with a wood filler, or to a floor that has been shellacked or varnished. Our Letter Circular 275, on "Polishes", gives information on, and formulas for, floor polishes or waxes. This will be sent free upon request. 
LC 339, Floor Oils -2 .

Information on the care and treatment of wood floors will be found in the book "The Expert Wood Finisher" by

A. Ashmun Kelly; published. by the Press of the Master Painter Publishing Company, Malvern, Pennsylvania.

U. S. Department of Agriculture Farmers' Bulletin 1219, "Floors and Floor Coverings", may be procured at five cents per copy (stamps not accepted) from the superintendent of Documents, Government Printing Office, Washington, D. C. 


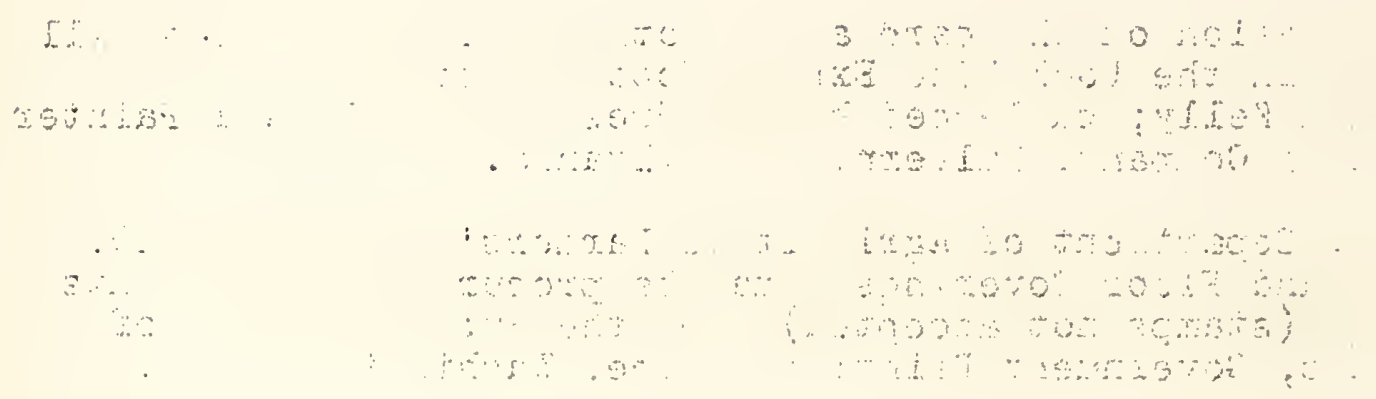



